\title{
Synthesis and Characterization of a Carborane-containing Precursor for $\mathrm{B}_{4} \mathrm{C}$ Ceramics
}

\section{Dexuan Yan, Jiahui Chen, Yu Zhang, Yanzi Gou*}

Science and Technology on Advanced Ceramic Fibers and Composites Laboratory, National University of Defense Technology, Changsha, China

\author{
Email address: \\ y.gou2012@hotmail.com (Yanzi Gou) \\ ${ }^{*}$ Corresponding author
}

\section{To cite this article:}

Dexuan Yan, Jiahui Chen, Yu Zhang, Yanzi Gou. Synthesis and Characterization of a Carborane-containing Precursor for $\mathrm{B}_{4} \mathrm{C}$ Ceramics. Science Discovery. Vol. 9, No. 3, 2021, pp. 128-132. doi: 10.11648/j.sd.20210903.18

Received: April 15, 2021; Accepted: May 14, 2021; Published: May 24, 2021

\begin{abstract}
B}_{4} \mathrm{C}$ ceramic is one of widely used ceramics with properties of low density, high hardness, high thermal-stability and high neutron absorption cross-section. However, the high melting point and high hardness of $\mathrm{B}_{4} \mathrm{C}$ make it difficult to be prepared as materials with complex shape. Precursor derived method is an effective approach to prepare special-shaped ceramics. In the early research of $\mathrm{B}_{4} \mathrm{C}$ ceramic precursors, highly toxic and unstable decaborane was generally used as the boron source material, and precursors were also unstable and difficult to synthesize. In this work, a new $\mathrm{B}_{4} \mathrm{C}$ precursor is designed and synthesized, and m-carborane, which has stable chemical properties and high thermal stability, is used as the boron source. Firstly, m-carborane is converted into lithium salt after removing active hydrogen by n-butyllithium. Then, catalyzed by $\mathrm{Cu}(\mathrm{I})$, the lithium salt reacts with m-chloroiodobenzene so that monomer, bis(3-chlorophenyl)-m-carborane, is prepared. The monomer is polymerized through Ullmann Coupling reaction at the present of $\mathrm{Ni}(0)$ catalyst and Zinc powder, and precursor poly[bis(3-chlorophenyl)-m-carborane] is finally synthesized. The precursor is stable at room temperature in air and soluble in Tetrahydrofuran, with $\mathrm{Mw}$ of $1013 \mathrm{~g} / \mathrm{mol}$, and ceramic yield of $62.20 \%$ at $900^{\circ} \mathrm{C}$ in $\mathrm{N}_{2}$. The precursor pyrolytic product is composed of $\mathrm{B}_{4} \mathrm{C}$ and graphite after heat treated at $1600^{\circ} \mathrm{C}$ in inert atmosphere.
\end{abstract}

Keywords: Precursor, Carborane, $\mathrm{B}_{4} \mathrm{C}$

\section{一种含间碳硼烷基团的碳化硼陶瓷先驱体的合成与表征}

闰德轩, 陈家辉, 张禹, 苟燕子*

国防科技大学, 新型陶瓷纤维及其复合材料重点实验室, 长沙, 中国

邮箱

y.gou2012@hotmail.com (苟燕子)

摘要：碳化确陶瓷具有密度低、硬度高、热稳定性好和中子吸收截面高的特性, 但是其高熔点、高硬度的特性也使得 其难以制备成具有复杂形状的材料。而先驱体转化法是制备复杂形状的陶瓷材料的重要方法。早期的碳化硼陶瓷先驱 体合成普遍以剧毒且不稳定的十硼烷为硼源物质, 合成难度高, 稳定性差。本文以化学性质稳定、热稳定性高的间碳

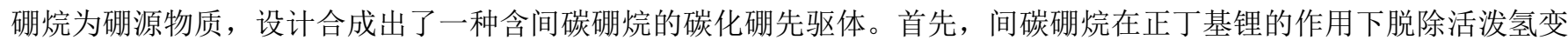
为碳硓烷锂盐, 再在亚铜离子的催化下与间氯碘苯反应生成双（3-氯苯基）间碳嗍烷单体。然后，单体在 $\mathrm{Ni}(0)$ 催化体 系和锌粉还原剂的还原催化下发生Ullmann偶联聚合, 得到先驱体---聚[双(3-氯苯基)间碳硼烷］(P3CB）。先驱体P3CB 为一种纯白色粉末状物质, 在水氧条件下稳定, 可溶解于四氢呋喃, 其重均分子量为 $1013 \mathrm{~g} / \mathrm{mol}$, 在氮气中 $900^{\circ} \mathrm{C}$ 陶瓷 产率为 $62.20 \%$ 。先驱体在惰性气氛中 $1600^{\circ} \mathrm{C}$ 无机化产物的物相组成为碳化硼和石墨。 
关键词: 先驱体, 碳硼烷, 碳化硼

\section{1. 引言}

碳硼烷（carborane）是一类以碳原子和嗍原子为骨架 的类硼烷化合物。其中犹以具有闭合二十面体结构的二碳 十硼烷 (dicarbadodecaborane, $\mathrm{C}_{2} \mathrm{~B}_{10} \mathrm{H}_{12}$ ) 最具特色, 三维 立体的超芳香性赋予了其优异的热稳定性、化学稳定性和 丰富的化学可修饰性[1]。因其性质特殊, 研究的丰富性远 超其他同类化合物, 因此如无特指, 本文均将二碳十硼烷 简称为碳硼烷。根据两个碳原子在碳硼烷笼状结构中位置 的不同, 碳硼烷具有三种同分异构体：邻碳嗍烷 (o-carborane) 、间碳嗍烷（ $m$-carborane）和对碳硼烷 ( $p$-carborane) , 且他们在高温下会发生重排转变。碳硼 烷于上个世纪 60 年代被发现后就得到了学界的广泛关注 [2-4], 其相关的衍生物和聚合物被大量地合成出来。聚合 物在引入碳硼烷基团后, 热稳定性、陶瓷产率等都有了显 著的提高, 如聚硅氧烷、聚苯炔、聚酰亚胺等 [5-11]。

先驱体转化法是一种极具优势的陶瓷材料制备方法, 利用先驱体聚合物易于分子设计、加工成型、陶瓷产率高 的特点, 能够实现原子级水平的元素混合以及复杂形貌陶 瓷材料的制备。碳化硼材料熔点高, 硬度、脆性大, 导致 烧结温度高, 机械加工困难, 因此, 制备出精密且结构复 杂的碳化硼材料更是难上加难。而先驱体转化法可以很好 的解决这一难题。前期众多关于碳化嗍先驱体的研究主要 集中在以十硼烷为硼源的合成路线上。虽然十硼烷的活性 高, 在高温下可以发生交联, 但是其化学性质不稳定, 对 水氧敏感，导致相应聚合物的合成困难，陶瓷产率也不高 [12-16]。碳硼烷虽然由十嗍烷合成而来, 但其作为一种稳 定的化合物, 却是“青出于蓝而胜于蓝”。尤其当碳硼烷作 为结构单元与其他共轭基团连接形成共轭聚合物时, 在热 稳定性和高陶瓷产率等方面展现出强大的优势。早在 1998 年Howard等人[17]就以 $\mathrm{Ni}(0)$ 催化的Ullmann偶联反应合 成出了聚[双(4-氯苯基)对碳硼烷]。这种聚合物虽然有着极 高的热稳定性和陶瓷产率, 但是其极高的分子链规整性使 其高度结晶并难溶于常见的多数溶剂, 这也使得其难以满 足陶瓷先驱体的应用需要。2017年Cheng等人 $[18,19]$ 以十 硼烷为初始物并同样经 $\mathrm{Ni}(0)$ 催化合成出了聚[双(4-氯苯基) 邻碳硼烷]。由于邻碳硼烷较对碳硼烷的对称性更低, 显 著降低了该聚合物的结晶性, 提高了其溶解性; 同时, 由 于该聚合物的主链也同样具有大共轭效应, 因而也能够达 到较高的陶瓷产率。

本文设计并合成出了溶解性良好的聚[双(3-氯苯基) 间碳嗍烷], 并对这种聚合物做了相应的表征和研究。

\section{2. 实验}

\section{1. 实验原料}

间碳硼烷购于郑州原理生物科技有限公司，纯度 $\geq 97 \%$ 。超干乙二醇二甲醚DME、超干四氢呋喃THF、1-
氯-3-碘苯、2,2'-联吡啶、双三苯基膦二氯化镍、三苯基膦、 锌粉（600目，99.99\%）、四乙基碘化铵、氞代氯仿、氛 代四氢呋喃均购于北京伊诺凯科技有限公司。正丁基锂 （1.6M正己烷溶液）、氯化亚铜购于上海麦克林生化科技 有限公司。吡啶购于西陇化工股份有限公司。

\section{2. 测试方法}

红外光谱 (FT-IR) 测试以 $\mathrm{KBr}$ 压片法在Perkin Elmer 公司Frontier红外光谱仪上进行。聚合物的核磁碳谱采用 Agilent公司的 400/54/ASP NMR核磁共振仪测量, 以氛代 氯仿为溶剂。聚合物的热稳定分析采用Perkin Elmer公司 的Pyris 1 TGA热重分析仪, 以氦气为载气, 升温速率为 $5^{\circ} \mathrm{C} / \mathrm{min}$ 。无机化产物的XRD物相分析以采用Bruker AXS 公司的D8 Advance X-射线衍射仪测量, 管电压 $40 \mathrm{KV}$, 管 电流 $40 \mathrm{~m} \mathrm{~A}$, 采用 $\mathrm{Cu} \alpha$ 靶 $(\lambda=0.154 \mathrm{~nm})$, 测量范围为 $2 \theta=10 \sim 90^{\circ}$ 。

\section{3. 双（3-氯苯基）间碳硼烷单体（3CB）的合成}

$250 \mathrm{ml}$ 四口烧瓶, 装有磁力搅拌磁子和橡胶翻口塞, 向反应瓶中加入 $2.884 \mathrm{~g}(0.02 \mathrm{~mol})$ 间碳硼烷。反应瓶抽 真空补氮气后, 通过注射器向反应瓶内注射入 $40 \mathrm{ml}$ 超干乙 二醇二甲醚DME, 并搅拌使原料全部溶解。在冰水浴条件 下, 通过注射器向反应瓶内逐滴加入 $21 \mathrm{ml}(0.042 \mathrm{~mol})$ 浓 度为 $1.6 \mathrm{M}$ 的正丁基锂的正己烷溶液。在室温下反应 $15 \mathrm{~min}$ 后, 依次向反应瓶中加入 $5.2 \mathrm{~g}(0.052 \mathrm{~mol})$ 氯化亚铜、12 ml吡啶、 $10.014 \mathrm{~g}(0.042 \mathrm{~mol}) 1$-氯-3-碘苯。在 $95^{\circ} \mathrm{C}$ 下保 持回流搅拌 48 小时。反应结束后, 向冷却的反应溶液加入 约 $120 \mathrm{ml}$ 乙醚稀释并静置过夜。再将铜-吡啶沉淀过滤, 滤 渣由乙醚冲洗。将含有产物的乙醚滤液收集浓缩后, 沉入 稀盐酸中重结晶, 并再用去离子水冲洗三遍, 干燥后得到 粗产物。粗产物再经硅胶层析柱进行分离提纯, 洗脱剂为 正己烷, 计得产物 $5.4 \mathrm{~g}$, 产率约 $74 \%$ 。FT-IR $\left(\mathrm{KBr}, \mathrm{cm}^{-1}\right)$ : 2612、2590 (B-H);1594、1571、1479 (Ar-H) ;1105、 1076 (B-B) ;726 (C-B) ; 681（m- $\mathrm{C}_{6} \mathrm{H}_{4}$ o.o.p.)。

\section{4. 聚[双(3-氯苯基)间碳嗍烷]（P3CB）的合成}

$250 \mathrm{ml}$ 三口烧瓶, 装有磁力搅拌磁子和橡胶翻口塞, 向 反应瓶中加入 $0.262 \mathrm{~g}(0.004 \mathrm{~mol})$ 的双三苯基膦二氯化镍、 $0.210 \mathrm{~g}(0.008 \mathrm{~mol})$ 的三苯基膦、 $0.210 \mathrm{~g}(0.008 \mathrm{~mol})$ 的四乙 基碘化铵、 $0.063 \mathrm{~g}(0.004 \mathrm{~mol})$ 的 $2,2^{\prime}$-联吡啶、 $0.915 \mathrm{~g}(0.014 \mathrm{~mol})$ 的锌粉。反应瓶抽真空补氮气后, 通过注射器向反应瓶内注 射入 $15 \mathrm{ml}$ 超干四氢呋喃 $\mathrm{THF}$ 。在 $60^{\circ} \mathrm{C}$ 反应约 10 分钟后, 将 $1.095 \mathrm{~g}(0.003 \mathrm{~mol})$ 双 (4-氯苯基) 间碳硼烷加入反应瓶中。 保持 $60^{\circ} \mathrm{C}$ 搅拌回流 24 小时。反应停止后, 向冷却的反应液中 加入约 $100 \mathrm{ml} \mathrm{HCl}$ /甲醇 $(2: 8)$ 溶液, 充分搅拌以除去未反 应完的锌粉。将混合液中的白色析出物离心分离后, 再用热 甲醇离心清洗三遍以上。将得到的粗产物溶于四氢呋喃THF 后, 再次沉入甲醇中再结晶。提纯后的产物再经 $80^{\circ} \mathrm{C}$ 真空干 
燥12小时, 计得纯白色产物0.8g, 产率约91\%。FT-IR ( KBr, $\left.\mathrm{cm}^{-1}\right)$ : 2604 (B-H);1598、1574、1476 (Ar-H);1086 (B-B);728 (C-B) ; 697 (m- $\mathrm{C}_{6} \mathrm{H}_{4}$ o.o.p.) 。

\section{3. 结果与讨论}

\section{1. 聚[双(3-氯苯基)间碳硼烷（P3CB）的合成与表征}

聚[双(3-氯苯基)间碳硼烷] ( $\mathrm{P} 3 \mathrm{CB}$ ) 的合成路线及其光 学照片如图1所示。 $\mathrm{P} 3 \mathrm{CB}$ 在室温下为纯白色粉末状物质, 对水氧不敏感, 可以长期保存在室温空气环境下。为了表 征和分析双（3-氯苯基）间碳硼烷单体 (3CB) 和聚[双(3氯苯基)间碳嗍烷] (P3CB) 的化学结构, 分别对其进行了 红外光谱 (FT-IR)、核磁碳谱 $\left(\mathrm{NMR}-{ }^{13} \mathrm{C}\right)$ 和凝胶渗透色 谱 (GPC) 的分析表征。其红外光谱如图2所示, 可以看到, 由于P3CB和其单体 $3 \mathrm{CB}$ 的化学结构极为相似, 其红外吸收 光谱也相近。位于 $2600 \mathrm{~cm}^{-1}$ 附近的极为宽大的震动吸收峰 是间碳嗍烷的特征吸收峰。该峰其实是一系列 $\mathrm{B}-\mathrm{H}$ 键的震动 吸收峰, 由于碳化嗍烷基团的高度对称性, 这些B-H键化学
环境相近, 吸收峰位置也极其相近, 只有在较低浓度下的 红外光谱中才可以分辨。可以看到, 相比于单体 $3 \mathrm{CB}, \mathrm{P} 3 \mathrm{CB}$ 的间碳嗍烷特征峰发生了明显的红移, 其最大吸收波数从 $2612 \mathrm{~cm}^{-1}$ 处红移至 $2604 \mathrm{~cm}^{-1}$ 处。由诱导效应可知, 强吸电子 基团一般使振动吸收峰向高波数蓝移, 而共电子基团则反 之。又由结构分析和芳香性“休克尔规则”可知, 碳硼烷基 团是一种具有芳香性但缺电子的化学结构, 具有强烈的吸 电性。所以, $3 \mathrm{CB}$ 单体聚合后形成的联苯结构进一步向碳 硼烷基团贡献了电子云密度, 并使其特征吸收峰红移。这 一结构性的变化也在苯环的震动吸收峰上得到了印证。单 体3CB苯环上间位取代的特征峰位于 $681 \mathrm{~cm}^{-1}$ 处, 而其聚合 为 $\mathrm{P} 3 \mathrm{CB}$ 后该特征峰蓝移至 $697 \mathrm{~cm}^{-1}$ 处。这也说明了碳硼烷基 团的强吸电子性改变了苯环 (联苯) 结构上的电子云密度, 使得电子云密度平均化。值得注意的是, 在 $3000-2850 \mathrm{~cm}^{-1}$ 处还出现了甲基和亚甲基的振动吸收峰。这可能是在单体 合成过程中, 过量的正丁基锂与碳嗍烷锂盐在铜离子的存 在下发生了交叉偶联, 将正丁基引入到了碳嗍烷基团上。 所以, 在单体合成过程中要严格控制正丁基锂的加入量。

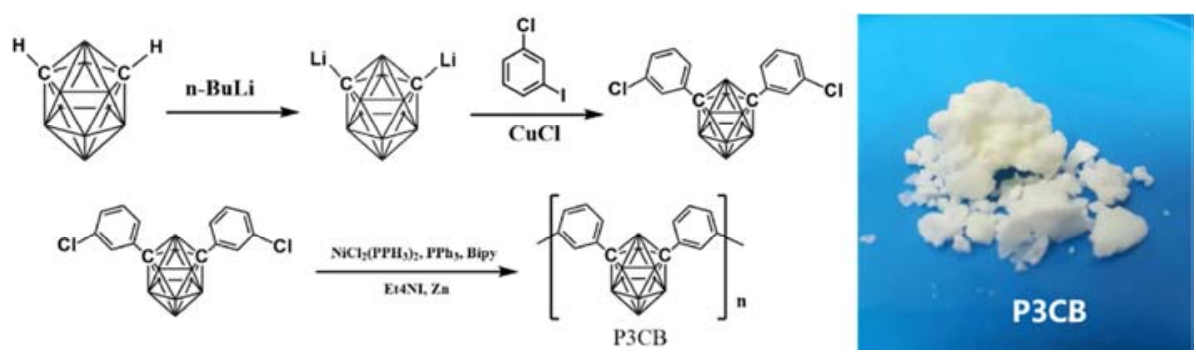

图1 $\mathrm{P} 3 \mathrm{CB}$ 及其单体 $3 \mathrm{CB}$ 的合成路线。

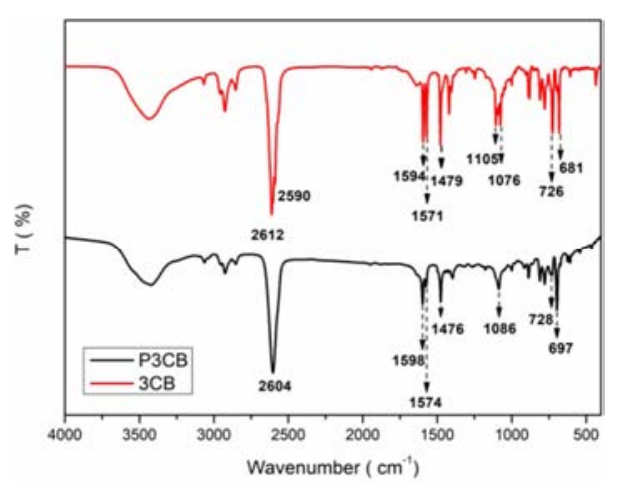

图2 $\mathrm{P} 3 \mathrm{CB}$ 及其单体 $3 \mathrm{CB}$ 的红外光谱。

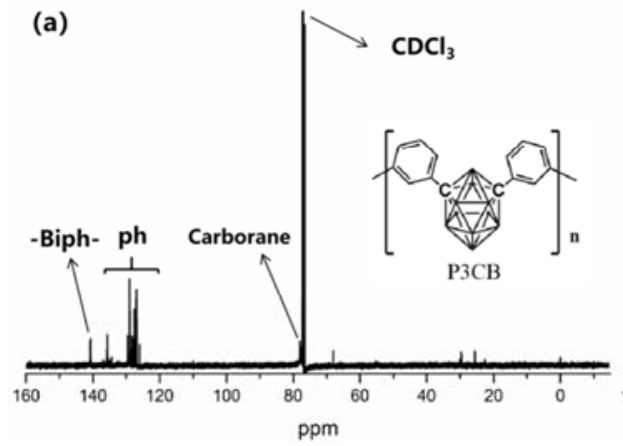

图3 P3CB（a）及其单体 $3 \mathrm{CB}$ （b）的核磁碳谱 ${ }^{13} \mathrm{C} N \mathrm{NMR}$ 。

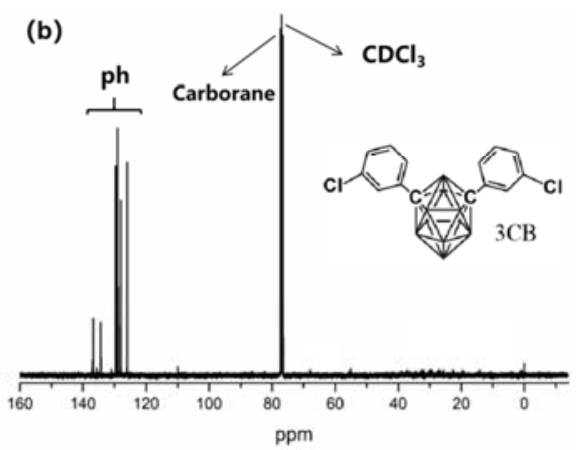

单体 $3 \mathrm{CB}$ 及 $\mathrm{P} 3 \mathrm{CB}$ 的核磁碳谱如图3所示。间碳硼烷上 两个碳原子的化学位移位于 $78 \mathrm{ppm}$ 附近, 与氛代氯仿的溶 剂峰极为接近。在 $3 \mathrm{CB}$ 聚合为 $\mathrm{P} 3 \mathrm{CB}$ 后, 碳硼烷上的碳原子 化学位移由 $77.18 \mathrm{ppm}$ 向高场移动至 $78.00 \mathrm{ppm}$ 处。在 126-136.8ppm之间的 6 个峰是3CB苯基上的 6 个不同化学环 境的碳原子的化学位移峰。而值得注意的是, 在 $3 \mathrm{CB}$ 聚合 为 $\mathrm{P} 3 \mathrm{CB}$ 后, 原本与氯原子相连的苯基碳原子的化学位移 移动到了 $140.74 \mathrm{ppm}$ 。而这一峰的出现, 也与联苯分子和 具有联苯结构聚合物的ph-ph键碳原子的特征峰相接近和 吻合 [19]。证明了3CB间发生了Ullmann偶联聚合, P3CB 中形成了联苯的结构单元。 
$\mathrm{P} 3 \mathrm{CB}$ 的凝胶色谱及分子量分布如图4所示。 $\mathrm{P} 3 \mathrm{CB}$ 的 数均分子量为 $599 \mathrm{~g} / \mathrm{mol}$, 重均分子量为 $1013 \mathrm{~g} / \mathrm{mol}$, 分子量 分布指数为 1.69 。这说明 $\mathrm{P} 3 \mathrm{CB}$ 的聚合度为 $2 \sim 3$, 属于一种 寡聚物。 $\mathrm{P} 3 \mathrm{CB}$ 的分子量较低很可能是受制于空间位阻效 应。由于单体 $3 \mathrm{CB}$ 的偶联反应位点的氯原子位于苯环的邻
位，其在相互作用时更易于受到体积更为巨大的碳化硼基 团的阻碍而难于聚合。同时，由于3CB中有少量的丁基取 代的氯苯基碳硼烷副产物, 该副产物也起到了封端剂的作 用。但是较低的分子量也使得 $\mathrm{P} 3 \mathrm{CB}$ 具有较好的溶解性, 使得其可以溶解于四氢呋喃和乙醚等中等极性溶剂。

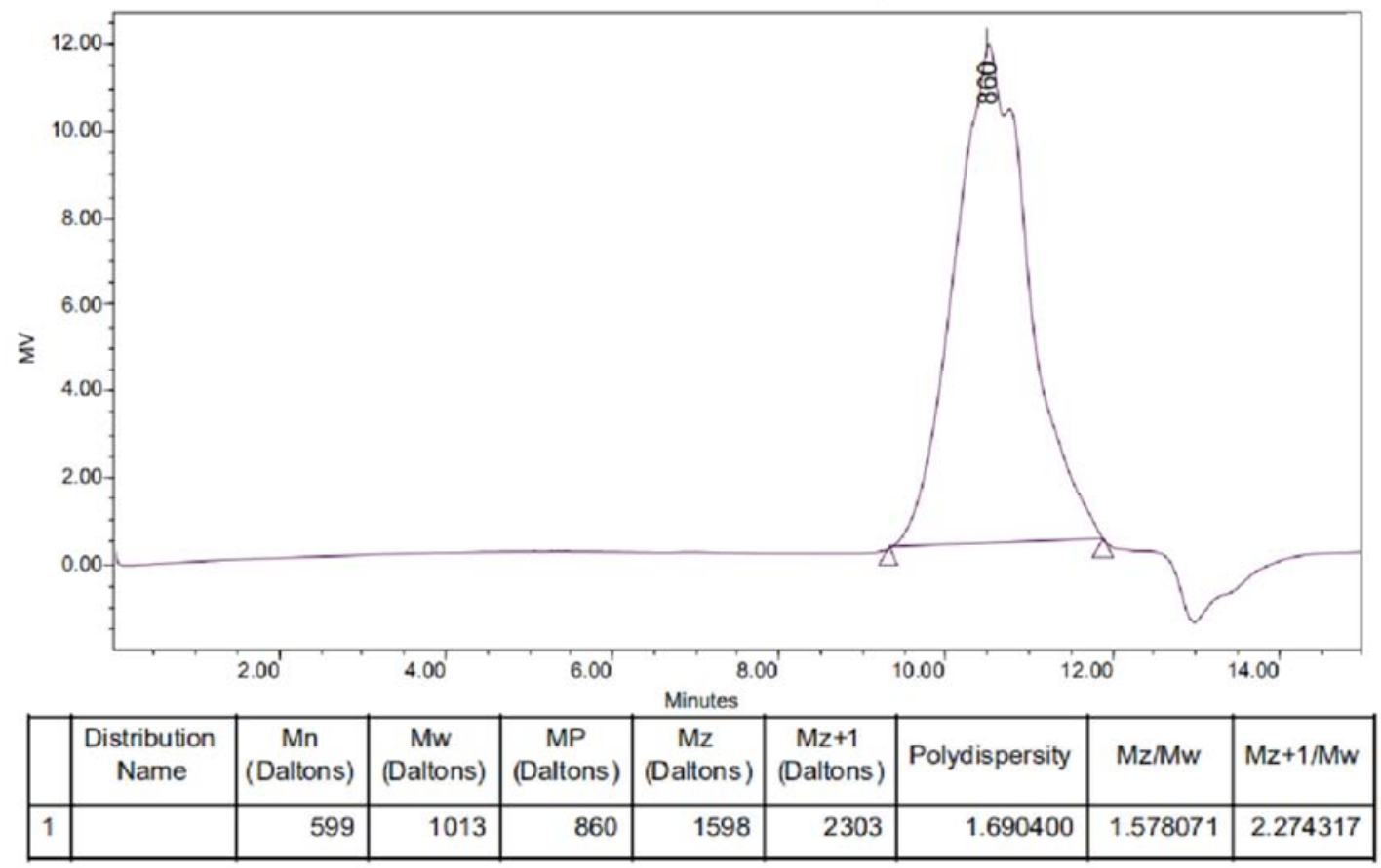

图4 P3CB的GPC色谱图和分子量分布。

3.2. 聚[双(3-氯苯基)间碳嗍烷]（P3CB）的无机化转变研 究

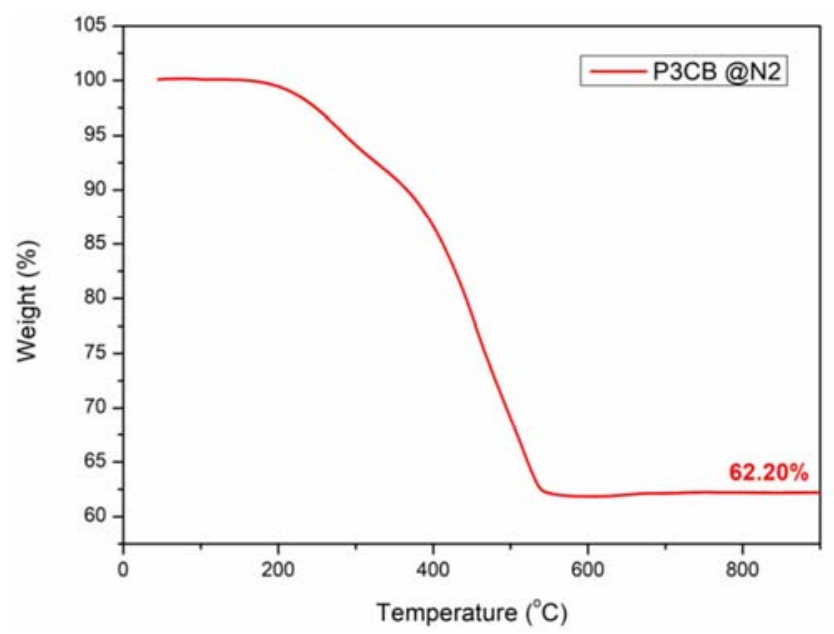

图5 P3CB在氮气气氛下的热重曲线。

$\mathrm{P} 3 \mathrm{CB}$ 的热重曲线如图5所示。可以看到, $\mathrm{P} 3 \mathrm{CB}$ 在氮气 气氛下 $900^{\circ} \mathrm{C}$ 的陶瓷产率到达了 $62.20 \%$ 。在其无机化过程中 主要存在两个失重过程。第一失重阶段为 $200-400^{\circ} \mathrm{C}$, 第二 失重阶段为 $400-550^{\circ} \mathrm{C}$ 。而在 $550^{\circ} \mathrm{C}$ 后, 物质已无明显的失 重, 无机化转变完全。而P $3 \mathrm{CB}$ 在不同温度下保温一个小时 后的无机化产物的XRD图如图6所示。可以看到 $\mathrm{P} 3 \mathrm{CB}$ 在 $1100^{\circ} \mathrm{C}$ 保温一小时后的无机化产物虽然已经有碳化嗍特征 峰的出现, 但是特征峰强度较低, 结晶度也较低。在 $1200^{\circ} \mathrm{C}$ 处理后, $\mathrm{P} 3 \mathrm{CB}$ 无机化产物的碳化硼结晶度提高, 但同时也 有石墨特征峰的出现。石墨相的出现显然与P3CB本征的化 学组成有关。 $\mathrm{P} 3 \mathrm{CB}$ 的硼碳元素比为7: 5, 远低于碳化硼的 4: 1 的硼碳比, 所以会有残余碳在P $3 \mathrm{CB}$ 的无机化产物中, 并在高温下形成石墨相。这一现象也与类似结构的聚[双(3氯苯基)间碳硼烷]的无机化转变相似 [19]。

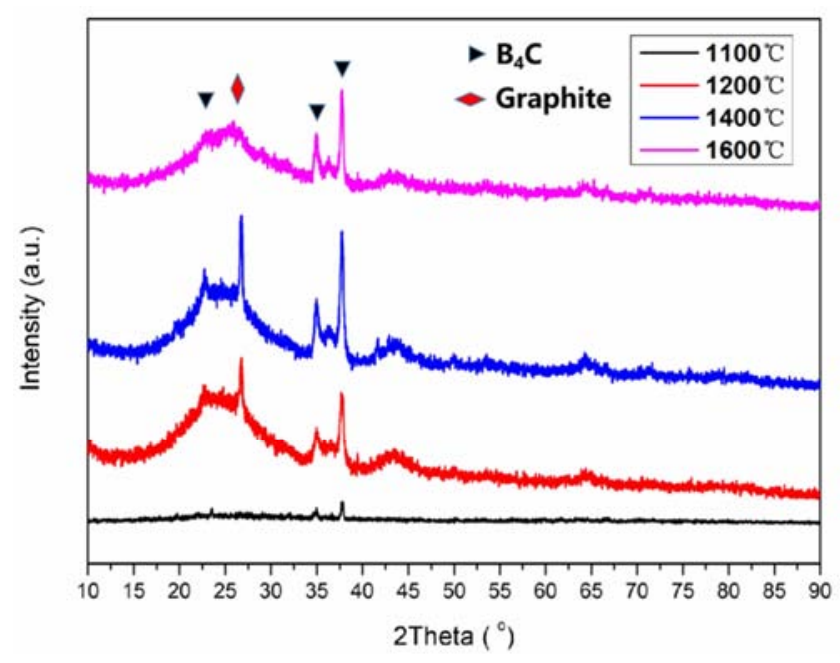

图6 P3CB在不同温度下无机化产物的XRD。 


\section{4. 结论}

本文合成和表征了一种含碳硼烷基团的碳化嗍先驱 体---聚[双(3-氯苯基)间碳硼烷]（P3CB），并研究了其化 学结构和无机化过程。首先, 由间碳硼烷在正丁基锂和亚 铜盐的催化下与间氯碘苯反应制备得到了单体---双（3-氯 苯基) 间碳硼烷 $3 \mathrm{CB}$ 。再在镍 (0) 催化下, 3CB进行Ullmann 偶联聚合得到了 $\mathrm{P} 3 \mathrm{CB}$ 。 $\mathrm{P} 3 \mathrm{CB}$ 为一种纯白色粉末状物质, 在水氧条件下稳定, 可溶解于四氢呋喃。由于空间位阻效 应的影响, $\mathrm{P} 3 \mathrm{CB}$ 先驱体的分子量较低, 数均分子量为 $599 \mathrm{~g} / \mathrm{mol}$, 重均分子量为 $1013 \mathrm{~g} / \mathrm{mol}$, 属于一种寡聚物。先 驱体在惰性气氛下 $900^{\circ} \mathrm{C}$ 的陶瓷产率为 $62.20 \%, 1600^{\circ} \mathrm{C}$ 无 机化产物主要为碳化嗍和石墨。P3CB可用做碳化䃉陶瓷 先驱体。同时也因其富嗍的性质，也可以吸收热中子而应 用于核防护领域。

\section{致谢}

本文为国家自然科学基金面上项目 (编号: 51772327) 的阶段性成果之一。

\section{参考文献}

[1] Scholz M, Hey-Hawkins E. Carbaboranes as pharmacophores: properties, synthesis, and application strategies [J]. Chemical Reviews, 2011, 111(11): 7035-7062.

[2] Fein M M, Grafstein D, Paustian J E, et al. Carboranes. II. The Preparation of 1- and 1,2-Substituted Carboranes [J]. Inorganic Chemistry, 1963, 2(6): 1115-1119.

[3] Hoffmann R, Lipscomb W N. Intramolecular Isomerization and Transformations in Carboranes and Substituted Polyhedral Molecules [J]. Inorganic Chemistry, 1963, 2(1): 231-232.

[4] Schroeder H, Vickers G D. The Structure of Neocarborane [J]. Inorganic Chemistry, 1963, 2(6): 1317-1319.

[5] Liu F, Fang G, Yang H, et al. Carborane-Containing Aromatic Polyimide Films with Ultrahigh Thermo-Oxidative Stability [J]. Polymers, 2019, 11(12): 1930-1943.

[6] Jiang $\mathrm{Y}, \mathrm{Li} \mathrm{X}$, Huang $\mathrm{F}$, et al. o-Carborane-Containing Poly(siloxane-arylacetylene)s With Thermal and Thermo-Oxidative Stabilities [J]. Journal of Macromolecular Science, Part A, 2015, 52(6): 476-484.
[7] Qi S, Han G, Wang H, et al. Synthesis and characterization of carborane bisphenol resol phenolic resins with ultrahigh char yield [J]. Chinese Journal of Polymer Science, 2015, 33(11): 1606-1617.

[8] Xing T, Zhang K. Syntheses of novel soluble carborane polyimides with ultrahigh thermal stability $[\mathrm{J}]$. Polymer International, 2015, 64(12): 1715-1721.

[9] Patel M, Swain A C, Cunningham J L, et al. The stability of poly $(m$-carborane-siloxane) elastomers exposed to heat and gamma radiation $[\mathrm{J}]$. Polymer Degradation \& Stability, 2006, 91(3): 548-554.

[10] Davis A R, Peterson J J, Carter K R. Effect of $o$-Carborane on the Optoelectronic and Device-Level Properties of Poly(fluorene)s [J]. 2012, 1(4): 469-472.

[11] Cansu-Ergun E G, Cihaner A. Electro-optical properties of poly[di(2-thiophenyl)carborane] and its opto-electronic application [J]. Materials Chemistry and Physics, 2013, 143(1): 387-392.

[12] Wang H, Wang J L, GOU Y Z. Progress of Advanced Boron Carbide Ceramic Materials Prepared by Precursor Derived Method [J]. Journal of Inorganic Materials, 2017, 32(8): 785-791.

[13] Xiaojuan Zhang, Jing Li, Ke Cao, et al. Synthesis and characterization of B-C polymer hollow microspheres from a new organodecaborane preceramic polymer [J]. Rsc Advances, 2015, 5:86214-86218.

[14] Pender, Mark, J., et al. Transition-Metal-Promoted Reactions of Boron Hydrides. 17. Titanium-Catalyzed [J]. Journal of the American Chemical Society, 2001, 123(49): 12222-12222.

[15] Pender M J, Sneddon L G. ChemInform Abstract: An Efficient Template Synthesis of Aligned Boron Carbide Nanofibers Using a Single-Source Molecular Precursor [J]. Cheminform, 2000, 31(17): 280-283.

[16] Sneddon L G, Pender M J, Forsthoefel K M, et al. Design, syntheses and applications of chemical precursors to advanced ceramic materials in nanostructured forms [J]. Journal of the European Ceramic Society, 2005, 25(2-3): 91-97.

[17] Colquhoun H M, Herbertson P L, Wade K, et al. A Carborane-Based Analogue of Poly(p-phenylene) [J]. Macromolecules, 1998, 31(5): 1694-1696.

[18] Cheng S, Weng Z, Wang X, et al. Oxidative protection of carbon fibers with carborane-containing polymer $[\mathrm{J}]$. Corrosion Science, 2017, 127:59-69.

[19] Cheng S, Yuan K, Wang X, et al. Poly(phenylene-carborane) for boron-carbide/carbon ceramic precursor synthesized via nickel catalysis [J]. Polymer, 2017, 115: 224-231. 